 \\ UNIVERSITY OF \\ TECHNOLOGY
}

\section{Mathematical shape optimization of hip prosthesis design}

Citation for published version (APA):

Huiskes, H. W. J., \& Boeklagen, R. (1989). Mathematical shape optimization of hip prosthesis design. Journal of Biomechanics, 22(8-9), 793-804. https://doi.org/10.1016/0021-9290(89)90063-8

DOI:

10.1016/0021-9290(89)90063-8

Document status and date:

Published: 01/01/1989

\section{Document Version:}

Publisher's PDF, also known as Version of Record (includes final page, issue and volume numbers)

\section{Please check the document version of this publication:}

- A submitted manuscript is the version of the article upon submission and before peer-review. There can be important differences between the submitted version and the official published version of record. People interested in the research are advised to contact the author for the final version of the publication, or visit the $\mathrm{DOI}$ to the publisher's website.

- The final author version and the galley proof are versions of the publication after peer review.

- The final published version features the final layout of the paper including the volume, issue and page numbers.

Link to publication

\section{General rights}

Copyright and moral rights for the publications made accessible in the public portal are retained by the authors and/or other copyright owners and it is a condition of accessing publications that users recognise and abide by the legal requirements associated with these rights.

- Users may download and print one copy of any publication from the public portal for the purpose of private study or research.

- You may not further distribute the material or use it for any profit-making activity or commercial gain

- You may freely distribute the URL identifying the publication in the public portal.

If the publication is distributed under the terms of Article 25fa of the Dutch Copyright Act, indicated by the "Taverne" license above, please follow below link for the End User Agreement:

www.tue.nl/taverne

Take down policy

If you believe that this document breaches copyright please contact us at:

openaccess@tue.nl

providing details and we will investigate your claim. 


\title{
MATHEMATICAL SHAPE OPTIMIZATION OF HIP PROSTHESIS DESIGN
}

\author{
R. Huiskes and R. BoEkLAGEN \\ Biomechanics Section, Institute of Orthopaedics, University of Nijmegen, P.O. Box 9101, $6500 \mathrm{HB}$ \\ Nijmegen, The Netherlands
}

\begin{abstract}
The long-term success of artificial-joint replacement depends partly on the chances for acrylic cement failure and interface distuption. These chances can be diminished by an optimal load-transfer mechanism, whereby stress concentrations are avoided. The present paper introduces a method for numerical shape optimization, whereby the finite element method is used iteratively to determine optimal prosthetic designs, which minimize interface stresses.

The method is first applied in a simplified one-dimensional model of a cemented femoral stem fixation, using acrylic cement. The results show that $30-70 \%$ cement and interface stress reductions can be obtained in principle with an optimized design. Although the actual optimal shape is susceptible to the characteristics of the joint load, the stem length, stem modulus, cement modulus and bone properties, its general geometrical characteristics are consistent, featuring proximal and distal tapers, and a belly-shaped middle region. These general characteristics are confirmed in a more realistic two-dimensional FEM model.

It is concluded that this method of shape optimization can provide a meaningful basis for prosthetic design and analysis activities in general.
\end{abstract}

\section{INTRODUCTION}

Artificial-joint replacement requires an endurable method of fixation, suitable to withstand the stresses generated by the loads for the longest possible period of time. The stress patterns in the bone-prosthesis structure depend on the magnitudes and orientations of the loads, the geometry of the structure, the mechanical (elastic) properties of the materials, and the physical conditions at material connections. The Finite Element Method (FEM) is a very suitable technique to interrelate these aspects quantitatively (Huiskes and Chao, 1983).

Traditionally, artificial-joint design improvement with the FEM has been a process of numerical 'trialand-error', either based on the creativity of the analyst, guided by FEM results, or based on systematic parametric variations in subsequent FEM calculations. Because the number of possible combinations of structural variations in a prosthetic design, even a simple femoral stem, is quite extensive, neither of these methods guarantees an optimal result. A better way to determine optimal design characteristics is by using numerical optimization methods.

Yang et al. (1984) introduced a numerical method, design sensitivity analysis, to be used in combination with the FEM, to facilitate the process of parametric analysis, and applied this technique in a preliminary study, analysing the dependence of cement strainenergy density levels on cement and stem elastic moduli in the femoral hip prosthesis. The present paper discusses the development and application of an FEM-based numerical method, suitable for mathematical shape optimization of prosthetic

Received in final form 29 July 1988 designs in general, using the femoral hip stem as an example.

Conceptually, this optimization method can be viewed in the following way. Traditionally, a specific prosthetic shape is taken as a basis for FEM analyses, by which the stress patterns in the bone-prosthesis structure are determined. Conversely, in the present case, some specific desired stress distribution is taken as a basis, and the FEM-optimization method is used to determine the corresponding design shape.

The present analysis was restricted to femoral prostheses, fixated with acrylic cement (PMMA), and the design objective was to minimize stress peaks in the cement and at the cement/bone interfaces, which are believed to be the prime causes for fixation failure (Huiskes, 1988). Because the process of numerical shape optimization is complicated and expensive, it was first applied to a simplified, general model of intramedullary fixation (Huiskes, 1980), in order to investigate its applicability, the trends of the solutions and their susceptibility to the various structural and loading parameters. In a second, two-dimensional FEM configuration, a more realistic optimal stem shape was determined.

\section{METHODS}

A global scheme for the FEM optimization procedure is shown in Fig. 1. The first two blocks (meshgenerator and FEM code) describe the FEM part, whereby stresses and strains in a model are determined based on descriptions of geometry (FE-mesh), external loads, material properties (elastic moduli, Poisson's ratios) and kinematic boundary conditions. In the present case, the MARC/MENTAT FEM system is used for this part (MARC Analysis Corporation, Palo Alto, CA, USA). 


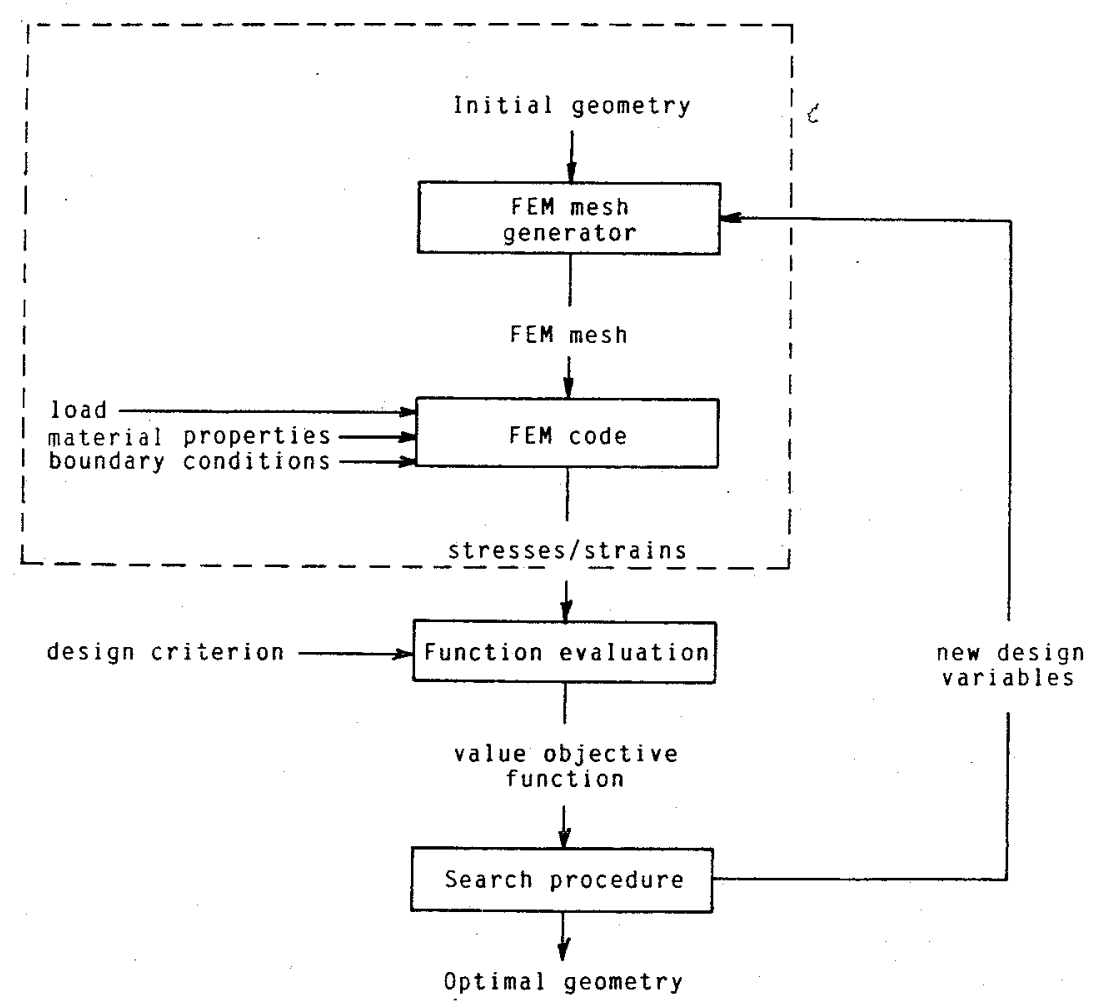

Fig. 1. A global scheme for the FEM numerical shape optimization procedure. The part within the dotted rectangle represents the MARC/MENTAT programs.

The geometry of the model is described by invariable geometric parameters, and by a number of design variables, suitable to represent the shape variations of the part to be optimized. These variables are described by the vector $\mathrm{v}=\left(v_{i}\right)^{T}, 1 \leqslant i \leqslant n$. Based on the design objectives (e.g., ninimal cement or interface stresses), an objective function $F$ is formulated, which depends on the stress, strain or strain-energy density (SED) distribution determined in the FEM evaluation. Because these quantities are functions of the design variables, $F=F(\mathrm{v})$. The value of this function is, in each iterative step, input to a search or optimization procedure, together with a number of geometrical constraints (e.g., limitation of the stem diameter to the width of the femoral canal), denoted by $a_{j} \leqslant f_{j}(\mathbf{v}) \leqslant b_{j}$, $1 \leqslant j \leqslant m$. The search procedure now minimizes

$$
\left\{F(\mathbf{v}) \mid a_{j} \leqslant f_{j}(\mathbf{v}) \leqslant b_{j}\right\}
$$

iteratively, to find the optimal set of design variables $\mathbf{v}=\mathbf{v}_{\boldsymbol{m}}$. For the first step, an initial set of design variables is selected.

The search procedure constitutes the core of the process, by which the objective function $F(v)$ is scanned for its minimal value. In this process, the combination of 'mesh-generator'-'FEM code'-'function evaluation' is addressed iteratively. For each set of design variables $\mathbf{v}_{k}$, the function $F\left(\mathbf{v}_{k}\right)$ is determined. The search procedure then consists of two parts. One, in which the search direction $\mathbf{s}_{k}$ over the $F(v)$ space is determined. For this purpose, approximations for the gradient are calculated by evaluating functional values $F\left(\mathbf{v}_{k}+\Delta\right)$ around $F\left(\mathbf{v}_{k}\right)$. In the second part, the objective function along the search direction $F\left(\mathbf{v}_{k}+t \mathbf{s}_{k}\right)$, $t \geqslant 0$, is evaluated to find its minimum for $t=\alpha$. The new set of design variables is then determined as $\mathbf{v}_{k+1}$ $=\mathbf{v}_{k}+\alpha \mathbf{s}_{k}$, and the process is restarted, until a minimal value $F\left(\mathbf{v}_{m}\right)$ is obtained. This value can be a local or an absolute minimum within the region of $F(v)$ bounded by the geometry constraints. To find the optimal set of design variables, different initial values can be assumed, each leading to a minimal set $\mathbf{v}_{m}$; the set for the smallest $F\left(\mathrm{v}_{m}\right)$ gives the optimal set.

Several iterative algorithms for the search procedure are available (e.g. Daniels, 1978). In the present program, a number of alternatives were implemented and used. These can be divided into simple search methods (simplex), slope-following methods (steepestdescent, conjugated gradient, least- $p$ th), and second order methods (Fletcher-Powell). Which of these is the most effective and efficient depends on the nature of the problem. During testing of these procedures it was found that the least-pth method usually gave the best results for the present problem. According to this method (Daniels, 1978), the objective function takes the form

$$
F(\mathbf{v})=\sum_{l=1}^{\beta}\left\{\omega_{l} e_{l}(\mathbf{v})\right\}^{p}
$$


where $e_{l}$ is a stress, strain, or SED related function, $\omega_{l}$ a weight factor, and in which the summation is over a number of relevant nodal points or integration points. In the case that $p=2$, this procedure is often called least-square optimization. The procedure further proceeds as a steepest-descent method, whereby the gradient of the objective function is used as a basis to change parameter values so as to proceed down the slope of the function most rapidly.

\section{$A$ simplified FE model}

Because of the complexity of the optimization procedure, the stem-design optimization method was applied first to a simplified, general model of intramedullary fixation (Huiskes, 1980), in order to investigate its applicability, solution trends, and the effects of loading and structural properties. The model consists of a bone cylinder, with uniform cross-section, containing an axisymmetric stem of variable thickness, cemented in the center (Fig. 2). The stem is loaded by an axial force $N(\mathrm{~N})$, a transverse force $T(\mathrm{~N})$, or a bending moment $M(\mathrm{~N} \mathrm{~mm})$. It was shown previously (Huiskes, 1980), that the mechanical behavior of this relatively simple structure can be characterized analytically by using beam-on-elastic-foundation theory, whereby the bone and the stem, separately, behave in accordance with linear-elastic beam theory. The acrylic cement layer, with its relatively low elastic modulus, behaves as a continuous row of linear-elastic springs (Winkler hypothesis), transferring the normal stresses (in bending) and the shear stresses (in axial loading) from the stem to the bone. The load-transfer mechanisms for the three different external loads are treated separately:

Axial force. The axial force is transferred through the cement layer by the axial shear-stress component $\tau_{r z}(r, z)$, which can be integrated per cross-section to the lumped axial shearing load or LSL, $q(z)\left(\mathrm{N} \mathrm{mm}^{-1}\right)$, by

$$
q(z)=\int_{0}^{2 \pi} \tau_{r z} r d \phi=2 \pi r \tau_{r z} .
$$

The axial rigidity of the cement layer per cross-section in the model, $C_{\mathrm{a}}(z)(\mathrm{MPa})$, is found from the Young's
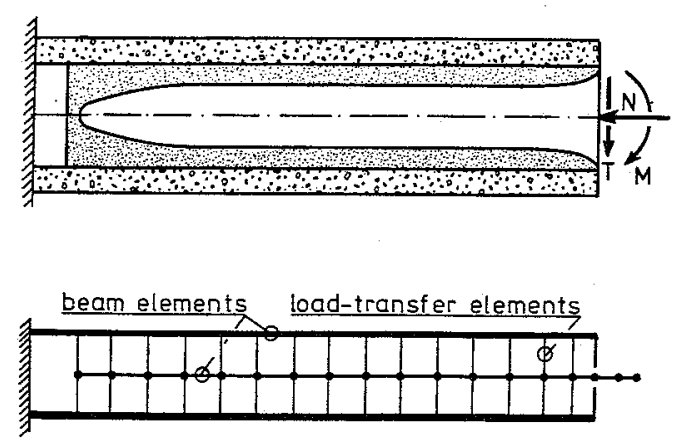

Fig. 2. Schematic characterization of the simplified, general model of intramedullary fixation (top) and the one-dimensional FEM representation (Huiskes, 1980). modulus and Poisson's ratio of the cement material, and the ratio between the local stem radius $r_{s}(z)(\mathrm{mm})$ and the inner bone radius $r_{\mathrm{b}}(\mathrm{mm})$ (Huiskes, 1980).

Bending and transverse force. The bending and transverse loads are transferred through the cement layer by the radial direct-stress component $\sigma_{r}(r, \phi, z)$ and the tangential shear-stress component $\tau_{r \phi}(r, \phi, z)$, which can be integrated per cross-section to obtain the lumped transverse load (LTL), $p(z)\left(\mathrm{Nmm}^{-1}\right)$, by

$$
p(z)=\int_{0}^{2 \pi}\left\{\sigma_{r} \cos \phi-\tau_{r \phi} \sin \phi\right\} r \mathrm{~d} \phi .
$$

The transverse cement layer stiffness per cross-section in the model, $C_{t}(z)(\mathrm{MPa})$, is found from the elastic modulus and Poisson's ratio of the cement material, and a function of the local stem radius $r_{s}(z)$ and the inner bone radius $r_{\mathrm{b}}$ (Huiskes and Schouten, 1980).

The FE-mesh for this simplified intramedullary fixation structure is shown in Fig. 2. The stem is described by 24 beam elements of variable stiffness properties, in accordance with the distributions of its axial and flexural rigidities. The bone is modeled by 24 beam elements of uniform stiffness properties, also in accordance with the values of its axial and fexural rigidities. The uniformity of the bone elements is, of course, no principle restriction in the FE schematization. The cement layer is modeled by 23 linear spring elements, in axial and transverse orientations, and stiffness values in accordance with the distributions of $C_{\mathrm{a}}(z)$ and $C_{\mathrm{t}}(z)$, respectively. This FEM beam model was tested earlier relative to three-dimensional (axisymmetric) continuum models (Huiskes, 1980).

\section{Application of the optimization technique}

The numerical shape optimization procedure was applied in combination with the above FE model, to optimize the longitudinal profile of the stem. This profile $r=r_{s}(z)$ was described by a restricted number of design variables $\mathrm{v}=\left(v_{i}\right)^{T}, 1 \leqslant i \leqslant n$, whereby $n$ was varied between 1 and 9 . The variables were expressed as $v_{i}=\arctan \left(\pi r_{i} / 2 r_{\mathrm{b}}\right)$, whereby $r_{i}(1 \leqslant i \leqslant n)$ is the stem radius, and $r_{\mathrm{b}}$ the inner bone radius. In this way the constrained design space $0 \leqslant r_{i} \leqslant r_{\mathrm{b}}$ (or $0 \leqslant \pi r_{i} / 2 r_{\mathrm{b}} \leqslant \pi / 2$ ) is transformed to a semi-infinite space for the design variables $v_{i}$.

The design objective was to minimize cement and cement/bone interface stress concentrations and hence to obtain uniform load-transfer from stem to bone, expressed as uniform distributions of the LSL, $q(z)$, and the LTL, $p(z)$. Uniform distributions for $q_{\mathrm{e}}(z)$ and $p_{\mathrm{e}}(z)$, whereby equilibrium can be obtained for the lowest possible maximal values, were determined theoretically using simple static equilibrium considerations.

In the FE schematization, the lumped internal loads $q(z)$ and $p(z)$ are described by the forces in the springs $f_{u i}$ and $f_{v i}$, whereby $q_{i}=2 f_{v i}\left(l_{i}+l_{i-1}\right)$ and $p_{i}=2 f_{u i} /$ $\left(l_{i}+l_{i-1}\right), 1 \leqslant i \leqslant \beta$, where $\beta$ is the number of springs, and $l_{i}$ the length of the beam elements (Fig. 2). The 
Table 1. Standard reference values and variations of the parameters used in the optimization procedures in the one-dimensional FEM model

\begin{tabular}{lcccc}
\hline Parameter/method & Symbol & Unit & Stand. ref. & Variations \\
\hline $\begin{array}{l}\text { No. of design } \\
\quad \text { variables }\left(r_{i}\right)\end{array}$ & $n$ & - & 9 & $1,2,3,5$ \\
Stem modulus & $E_{s}$ & $\mathrm{MPa}$ & $2 \times 10^{5}$ & $1.1 \times 10^{5}, 3 \times 10^{5}$ \\
Stem length & $L$ & $\mathrm{~mm}$ & 120 & 80,160 \\
Cement modulus & $E_{\mathrm{c}}$ & $\mathrm{MPa}$ & $2 \times 10^{3}$ & $1.5 \times 10^{3}, 2.5 \times 10^{3}$ \\
Bone modulus & $E_{\mathrm{b}}$ & $\mathrm{MPa}$ & $2 \times 10^{4}$ & $1.5 \times 10^{4}, 2.5 \times 10^{4}$ \\
Bone outer radius & $r_{\mathrm{o}}$ & $\mathrm{mm}$ & 15 & - \\
Bone inner radius & $r_{\mathrm{b}}$ & $\mathrm{mm}$ & 10 & - \\
\hline
\end{tabular}

objective functions $F(\mathbf{v})$ were formulated as, for axial loading

$$
F(\mathbf{v})=\sum_{i=1}^{\beta}\left(q_{i}(\mathbf{v})-q_{\mathrm{ei}}\right)^{2},
$$

and for transverse loading and bending

$$
F(\mathrm{v})=\sum_{i=1}^{\beta}\left(p_{i}(\mathrm{v})-p_{\mathrm{ei}}\right)^{2} .
$$

As described above, a new set of design variables $\mathbf{v}_{k}$ is determined after each iteration in the optimization procedure. From this set, the actual structural parameters are updated for each element, and the internal loads $q_{i}$ and $p_{i}$ are calculated in the FE program.

In order to develop an understanding of the relations between the design criterion (the objective function), the structural parameters, the optimization method and the design variables, an extensive parametric analysis was carried out. The optimization process was repeated for variations in the number of design variables, the form of the objective function, the optimization algorithm and the loading, geometry and material properties. Standard values and variations are shown in Table $1^{\circ}$

\section{Application to a two-dimensional FEM model}

In order to test whether the characteristics of the optimal shapes determined in the previous 'onedimensional' model would hold in principle for a more realistic configuration, the optimization procedure was applied in a two-dimensional model of the femoral bone-prosthesis structure. The FEM model (Fig. 3; plane-stress 4-node quadrilateral elements) used a side-plate element layer to account for the three-dimensional structural integrity of the bone. The bone geometry was based on an earlier study (Huiskes et al., 1981). The element thickness of the 'side-plate' and the 'front-plate' are non-uniform, and selected in such a way that the flexural rigidities of the stem and the bone are adequately described. The prosthesis was assumed to be loaded in pure bending.

As in the previous model, nine design variables were applied $(n=9)$, representing the stem width at nine equally spaced locations along the mid-stem axis. In the optimization procedure, the mid-stem axis (Fig. 3) was an invariable, and the nodal-point relocations

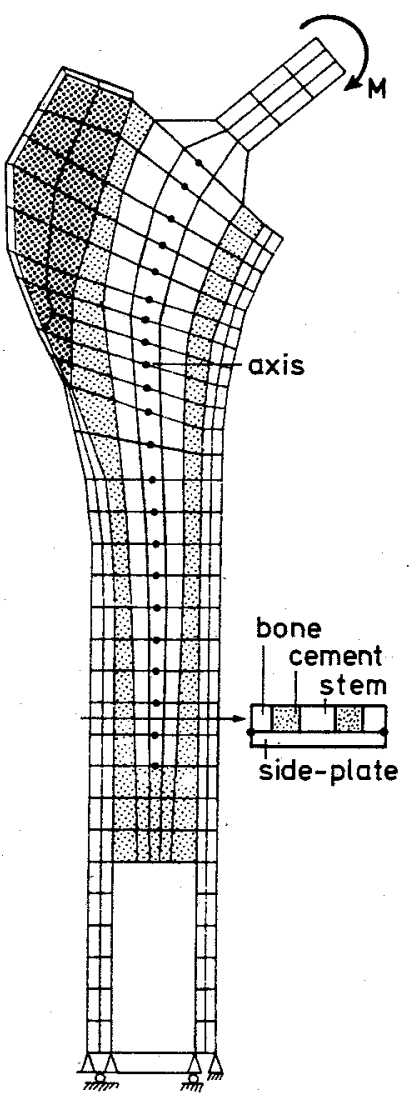

Fig. 3. Two-dimensional FEM model, featuring a side-plate and nonuniform element thickness distributions, used for a stem-shape optimization procedure. The different shades represent metal $\left(E=2.0 \times 10^{5} \mathrm{MPa}\right)$, acrylic cement $\left(E=2.0 \times 10^{3} \mathrm{MPa}\right)$, cancellous bone $\left(E=1.0 \times 10^{3} \mathrm{MPa}\right)$ and cortical bone $\left(\mathrm{E}=1.7 \times 10^{4} \mathrm{MPa}\right)$.

were determined from the design variables by linear interpolation. The boundary constraints, preventing the stem from becoming larger than the medullary canal width, were incorporated in the design variables by the same transformation as described in the previous section.

For the design objective, uniform load-transfer from stem to bone was selected. Because the optimal distribution of the stresses cannot be predicted analytically in this case, this objective was translated into a 
uniform distribution of the strain-energy density (SED) in the element integration points of the cement, along the cement/bone interface. It is expected that realization of this objective will yield uniform cement/bone interface stresses as well. The objective function takes the form

$$
F(\mathbf{v})=\sum_{k=1}^{\gamma}\left\{\omega_{k} U_{k}(\mathbf{v})\right\}^{p},
$$

where $U_{k}$ is the SED in the $k$ th integration point, and $\omega_{k}$ is a weight factor; $\omega_{k}=0$ at the utmost proximal/lateral side and under the stem tip, $\omega_{k}=2$ at the proximal/medial side, and $\omega_{k}=1$ elsewhere.

The least-pth optimization procedure was again applied, whereby $p$ was given different values. A high value of $p$ penalizes in particular the higher SED peaks, but reduces the rate of convergence. A compromise was found by increasing the value of $p$ in the course of the iterative process.

In recognition of the fact that FEM models of this kind are most useful when interpreted on a relative basis, the optimized stem shape was compared to traditionally shaped hip prostheses, analysed in the same two-dimensional FE model (Huiskes, 1988).

\section{RESULTS}

\section{Simplified model}

The number of design variables used presents a restraint to the general shape of the stem. When $n=1$, the stem is of uniform thickness, $n=2$ represents a straight taper (with the proximal and distal radii as the two design variables), $n=3$ a double taper, $n=5$ and $n=9$ more arbitrary shapes of (potentially) increasing complexity. When $n=1$, the objective function can be represented by a curve as a function of the (uniform) stem thickness. When $n=2$, the objective function can be shown as a plane, as it depends on the two determining radii. An example illustrating the optimization process in this case is shown in Fig. 4; from three different initial values of the two radii, the process converges to the optimal configuration of $r_{\text {distal }}=2.5 \mathrm{~mm}$ and $r_{\text {proximal }}=9 \mathrm{~mm}$.

The optimal stem shapes for $n=1, n=2, n=3$ and $n=9$ (transverse-force loading $T=100 \mathrm{~N}$ ) are shown in Fig. 5. The scale of the colors in the cement layer represent the values of the LTL, $p(z)$, transferred through the cement layer and the interfaces. The pattern of $p(z)$ in the case of the straight stem $(n=1)$ is

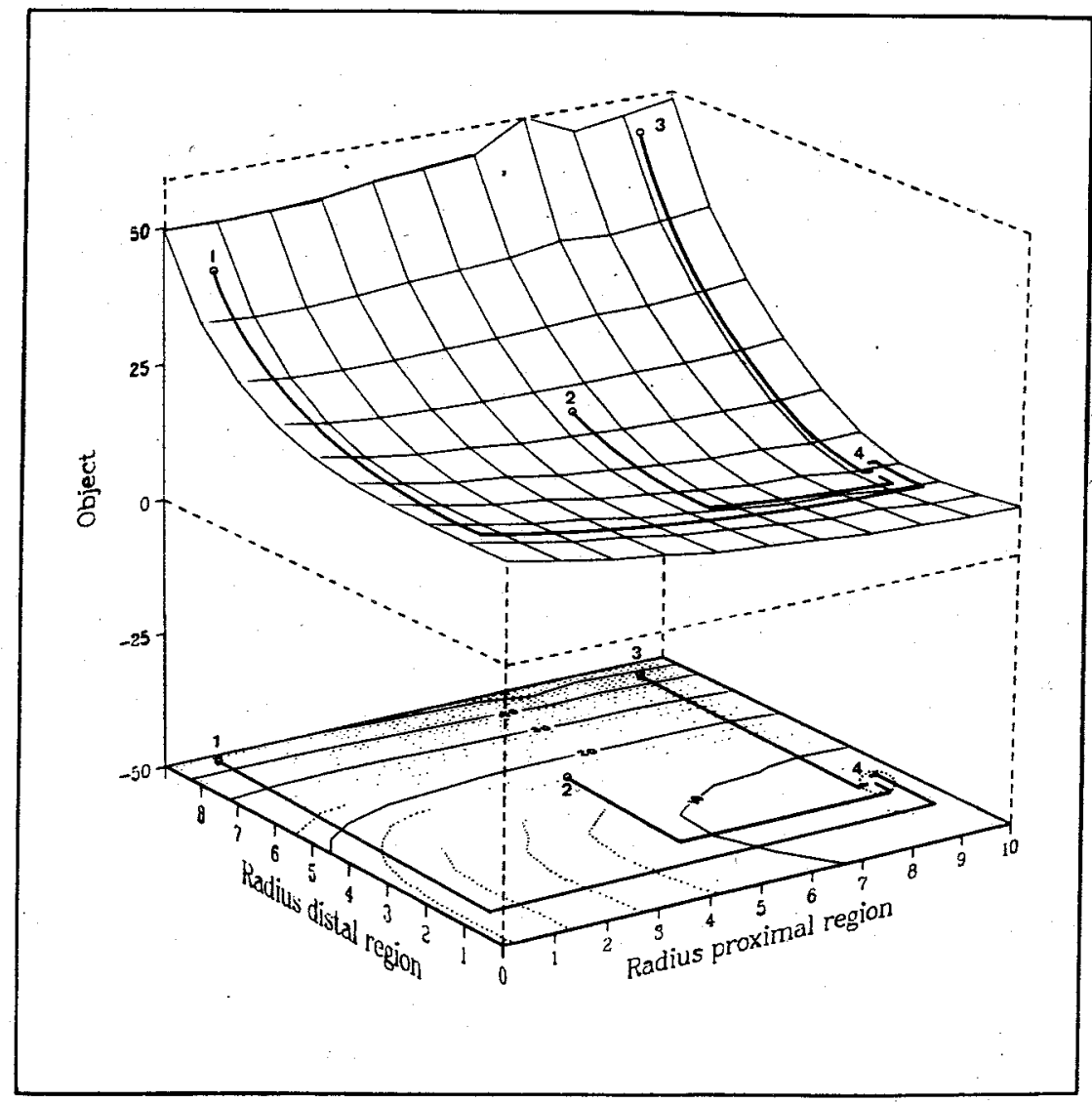

Fig. 4. Illustration of the optimization process in the simplified model for the case $n=2$ (proximal and distal stem radii as design variables). From alternative initial configurations $(1,2$ and 3$)$, the process converges to a minimum of the objective function (4). 
typical for this configuration, displaying peaks at the proximal and distal sides (Huiskes, 1980). The optimal stem radius is about $3.5 \mathrm{~mm}$, whereby the proximal and distal peak values in $p(z)$ are about 19 and $7.5 \mathrm{~N} \mathrm{~mm}^{-1}$, respectively. In the case of the straight taper $(n=2)$, a reduction of the distal peak to about $5 \mathrm{~N} \mathrm{~mm}^{-1}$ occurs, while the proximal peak reduces to $10 \mathrm{~N} \mathrm{~mm}^{-1}$. When $n=3$, the process converges to an overcut double taper, whereby the distal peak is further reduced to $2.8 \mathrm{~N} \mathrm{~mm}^{-1}$. The proximal peak reduces only slightly to $9.0 \mathrm{~N} \mathrm{~mm}^{-1}$. The optimal shape for the 'arbitrary' profile $(n=9)$ is characterized in particular by its proximal taper. The peak values of the LTL are now about $3.0 \mathrm{~N} \mathrm{~mm}^{-1}$ proximally and about $2.6 \mathrm{~N} \mathrm{~mm}^{-1}$ distally; hence, there is a reduction of peak cement and cement/bone interface loading of almost $85 \%$ relative to the straight stem, and $70 \%$ relative to the straight-tapered stem. In the final configuration, the course of $p(z)$ is very close to the shape which is theoretically optimal $\left(p_{\mathrm{e}}\right)$, as shown in Fig. 6(a).

A similar process evolves for the case that the stem is loaded by a bending moment $(M=100 \mathrm{~N} \mathrm{~mm})$. The application of nine design variables also results in a proximal taper, reducing the maximal proximal LTL peak from $0.7 \mathrm{~N} \mathrm{~mm}^{-1}$ in the straight-stem case to $0.3 \mathrm{~N} \mathrm{~mm}^{-1}$ for the 'arbitrary' profile with the proximal taper. Although a considerable reduction in the peak LTL occurs, the final course of $p(z)$ is not as ideal as in the case of transverse loading when compared to the theoretically optimal curve [Fig. 6(b)].

The optimized stem shape $(n=9)$ for axial-force


Fig. 6. Optimal stem profiles $(n=9)$ and associated distributions of the lumped transverse load (LTL) and the lumped shearing load (LSL) (continuous curves). The LTL, respectively LSL distributions for the straight-stem case (dotted curves) and the theoretically most optimal distributions ( $p_{\mathrm{e}}$ and $q_{\mathrm{e}}$, shaded areas) are shown as well. Note the differences in scales of the longitudinal and transverse stem dimensions. (a) Transverse force ( $T=100 \mathrm{~N}$ ); drastic LTL-peak reductions generated by the optimal stem profile, almost equal to the theoretically optimal LTL distribution. (b) Bending moment ( $M=100 \mathrm{Nmm}$ ); significant LTLpeak reductions generated by the optimal stem profile, but LTL distribution not as close to the theoretical one. (c) Axial force $(N=100 \mathrm{~N})$ : significant LSL-peak reductions generated by the optimal stem profile, almost equal to the theoretically optimal LSL distribution. 


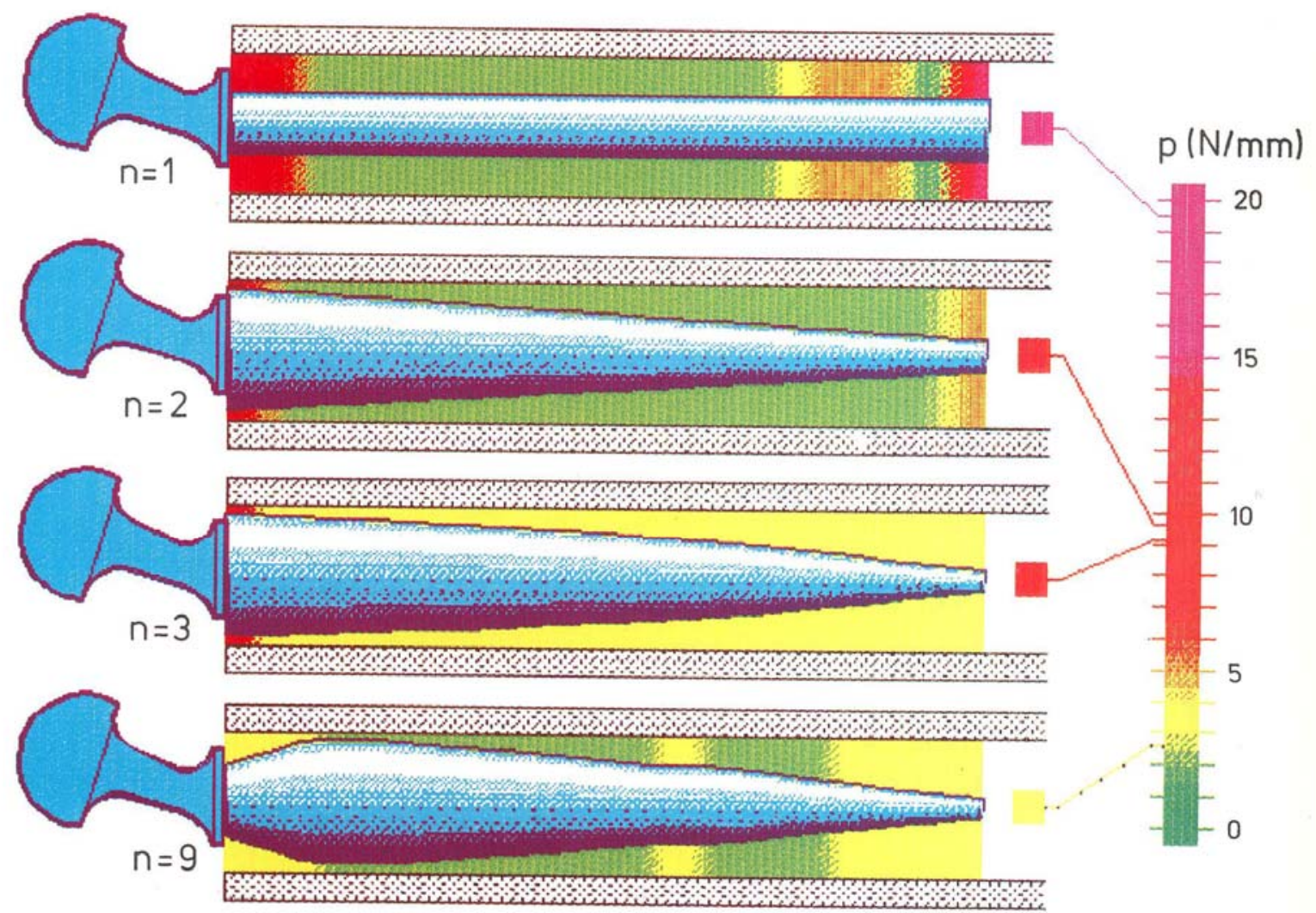

Fig. 5. Optimal stem shapes and distributions of the lumped transverse load (LTL) for increasing numbers of design variables: $n=1$ (uniform thickness); $n=2$ (straight taper); $n=3$ (dual taper); and $n=9$ ('arbitrary' profile). The maximal LTL value is indicated in each case (transverse force $T=100 \mathrm{~N}$ ). The arbitrary profile in particular, allows for an optimal stem shape whereby the cement and interface stress peaks are drastically reduced. 
loading $(N=100 \mathrm{~N})$ is somewhat different from those in the previous loading cases, in particular where its thickness is concerned [Fig. 6(c)]. The reduction in the LSL peaks is on the order of $40 \%$ relative to the straight stem and the tapered configurations. The course of the LSL $q(z)$ is almost equal to the theoretical objective $q_{\mathrm{e}}(z)$ [Fig. 6(c)].

The application of nine design variables in comparison to $n=5$ gives no large gain for the cases of axial and transverse-force loading. A significant gain is found in bending. In these models, convergence to the solution was monotonous; alternative initial shapes did not result in different solutions.

\section{Parametric analysis}

Parametric analysis were carried out relative to the stem length $(L)$, the stem modulus $\left(E_{\mathrm{s}}\right)$, the cement modulus $\left(E_{\mathrm{c}}\right)$ and the bone modulus $\left(E_{\mathrm{b}}\right)$. Each time, the complete optimization procedure was repeated for the case of nine design variables $(n=9)$ and transverse loading $(T)$.

The length was varied from $120 \mathrm{~mm}$ to $L=80 \mathrm{~mm}$ and $L=160 \mathrm{~mm}$. The optimal stem contour predicted for the shorter stem has less pronounced tapers at the proximal and distal sides [Fig. 7(a)]. At both ends, the maximal values of $p(z)$ are higher than for $L=120 \mathrm{~mm}$. When the length is increased to $L=160 \mathrm{~mm}$, the relative stem shape, drawn on the same length scale as the intermediate stem, is not very different from the reference case $(L=120 \mathrm{~mm})$. Although the LTL is generally lower, the proximal peak in $p(z)$ is higher for the longer stem [Figs 7(a) and 8].

Assuming titanium $\left(E_{\mathrm{s}}=1.1 \times 10^{5} \mathrm{MPa}\right)$ as stem material results in a similar optimal stem profile as for CoCr-alloy $\left(E_{\mathrm{s}}=2 \times 10^{5} \mathrm{MPa}\right)$. However, the proximal LTL peak is almost twice as high [Figs $7(\mathrm{~b})$ and 8$]$.

The assumption of a ceramic material $\left(E_{\mathrm{s}}=3\right.$ $\times 10^{5} \mathrm{MPa}$ ) results in a similar pattern of the LTL $p(z)$ as for $\mathrm{CoCr}$-alloy, with a slightly less pronounced proximal taper, and a somewhat thinner distal taper [Figs 7(b) and 8].

A variation of the acrylic cement modulus from $E_{\mathrm{c}}$ $=1500$ to $E_{\mathrm{c}}=2500 \mathrm{MPa}$ results in only marginal differences in the distribution and the maximal values
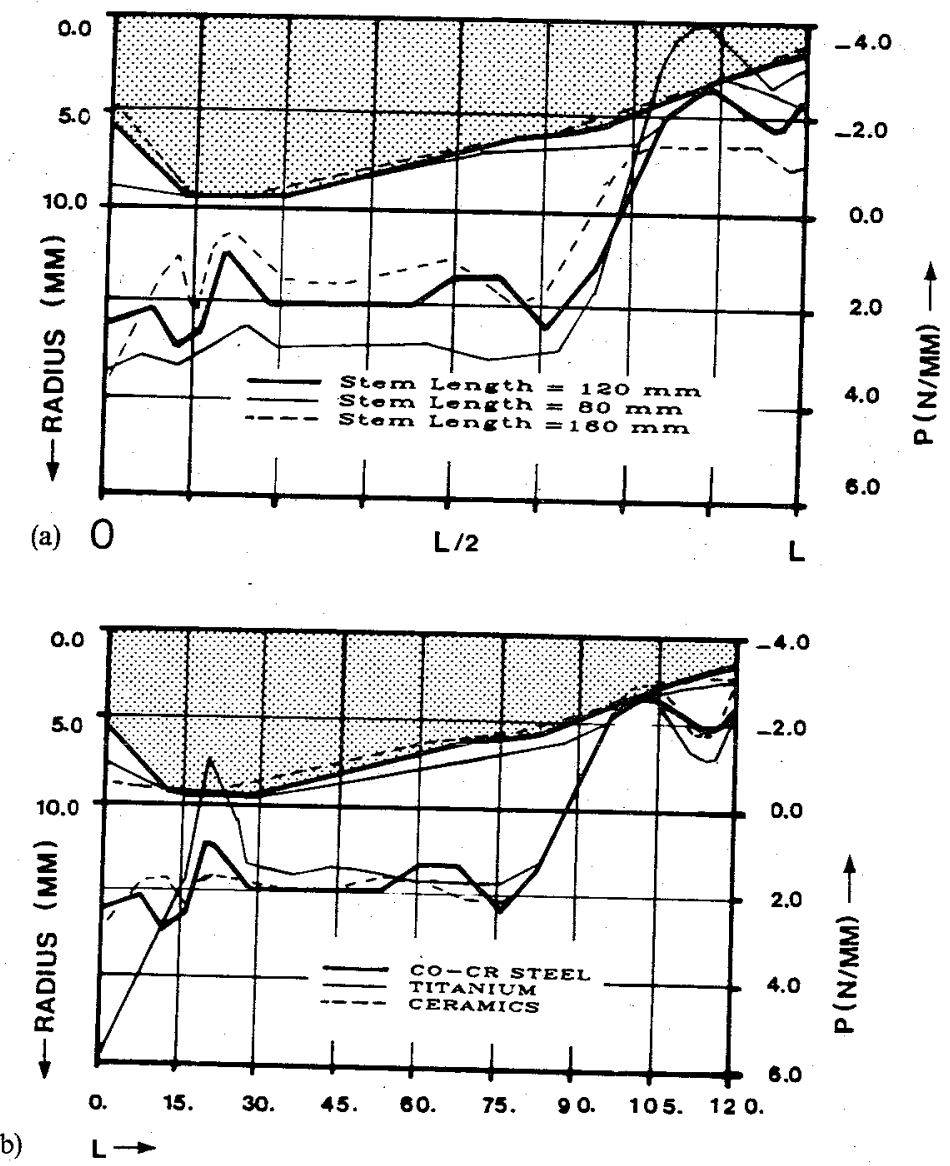

Fig. 7. Results of the parametric analysis, whereby the stem length (a) and the stem elastic modulus (b) were varied. For each case the optimal stem profile is shown (note the differences in longitudinal and transverse scales), and the associated distributions of the lumped transverse load, LTL (transverse force $T=100 \mathrm{~N}$, $n=9$ ). 
of $p(z)$. The optimal stem profiles are almost equal; however, in the case of the lowest modulus, a less pronounced proximal taper results (Fig. 8).

A variation of the bone modulus between $E_{\mathrm{b}}=15,000$ and $E_{\mathrm{b}}=25,000 \mathrm{MPa}$ also results in similar LTL patterns and only slightly different maximal values of $p(z)$. More difference occurs in the profiles of the optimal stems; the higher the bone modulus, the more pronounced the proximal taper (Fig. 8).

\section{Two-dimensional FEM model}

The optimized profile of the prosthesis in the twodimensional FEM model, and the SED it generates in the cement near the cement/bone interface, are shown in Fig. 9 (a), and compared to the corresponding data for a traditionally shaped stem in Fig. 9 (b). The latter model had the most favorable cement and interface stress patterns of six stem types in a comparative stress analysis using a similar two-dimensional FEM model (Huiskes, 1988). It must be noted that in the FEM analysis of the traditional prosthesis, the prosthetic collar was assumed to have no contact with the calcar bone. The comparison shows that the SED peaks at the proximal and distal sides (typical for intramedullary hip prostheses in general) have diminished for the optimal shape. The highest values now occur on the distal side, under the tip of the stem, representing predominantly the longitudinal stresses in the cement due to bending of the diaphysis. The reduction in SED peaks in the cement is accompanied by reduced cement/bone interface shear and normal stresses, together indicating that cement failure and interface disruptions are less likely.

Evidently, the general characteristics of the optimized shapes determined in the simplified onedimensional model, featuring proximal and distal


Fig. 8. Proximal radii of the optimal stem profiles, and the associated peak values of the lumped transverse load, LTL, found with the optimization procedure for variable stem length, and stem, cement and bone moduli (transverse force $T$ $=100 \mathrm{~N}, n=9$ ). Except for the titanium stem material $(E$ $=1.1 \times 10^{5} \mathrm{MPa}$ ), drastic reductions of the LTL peaks, almost to the theoretically optimal, are obtained in all cases. However, the actual optimal shapes (in particular the proximal tapers) differ somewhat.



(a)

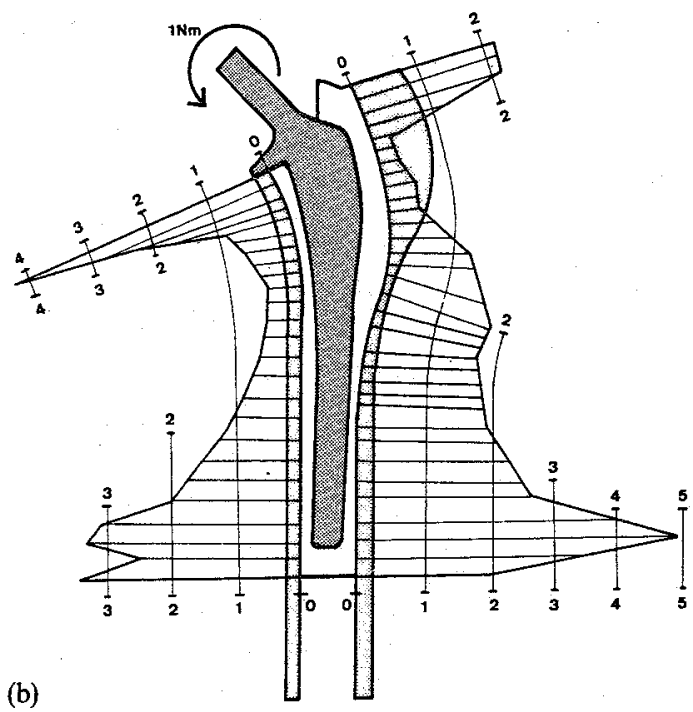

Fig. 9. (a) Optimal stem shape and associated SED distribution in the cement, as result of the optimization procedure in the two-dimensional FEM model (SED $\times 10^{-13} \mathrm{~N} \mathrm{~mm}^{-2}$ ). (b) Stem shape and associated SED distribution (SED $\times 10^{-13} \mathrm{Nmm}^{-2}$ ) in the cement around a traditionally shaped stem. Note that no collar/calcar contact was assumed in this analysis. Proximal and distal SED-peaks are found in this case, due to the load-transfer mechanism. These peaks are absent when the stem shape is optimized (a).

tapers, and a belly-shaped middle region, are reproduced in the more realistic two-dimensional model.

\section{DISCUSSION}

Relative to the design optimization methods presented here, it must be appreciated that 'to err is 
human, but to really foul things up requires a computer' (source unknown). The replacement of human creativity by a mathematical computer procedure does not solve all problems of artificial-joint design in a general sense, but rather brings them into focus, whereby they seem to have grown.

The most important of these problems is related to the optimization objective, the structural failure mode relative to which the stress patterns are optimized. Although it is evident that the most important longterm clinical problem of artificial-joint fixation is loosening (Stauffer, 1982), it is not entirely certain how the long-term clinical failure mechanisms are related to the mechanical characteristics in the idealized, immediate post-operative configuration. Apart from purely mechanical failure phenomena such as yield and fracture, biomechanical reactions of bone to stresses, strains and relative motions play a role here too (Huiskes, 1986). Probable initial sources of eventual clinical failure which can be identified are: (i) disruption of material connections (e.g. cement/ bone and cement/implant, or implant/bone interfaces); (ii) fracture of acrylic cement; (iii) fracture of prosthetic components; (iv) strain-related ('stress-shielding') adaptations of bone mass and structure; and ( $v$ ) resorption of bone at implant/bone interfaces due to relative motions once the interface is disrupted. Objective functions based on minimizing the chances for each of these phenomena to occur can be formulated. In principle, however, each of these criteria may lead to a different optimal design.

The second problem is related to the external load for which the design characteristics are optimized. The joint load is one variable in a multitude of functions, and each particular loading system leads to different stress patterns, and hence may result in a different optimal prosthetic shape. Typically, a force resembling that in the limb support phase of gait is assumed in FEM analyses of hip arthroplasty (Crowninshield et al., 1978). However, it is probable that high hip-joint forces of a different orientation are also generated in functions other than level gait (Davy et al., 1988).

The bending component of the hip-joint load in particular generates the highest stress peaks in the load-transfer mechanism (Huiskes, 1986). Pure bending is attractive as a test load for comparative purposes, because its effects do not depend on a point of application or an orientation. In addition, pure bending can be considered as a 'substitute' load for a number of loading cases out of a 'daily' cycle (Huiskes et al., 1987).

A third problem is the bone, which varies to some extent in shape, dimensions and material properties in different individuals. Evidently, the optimal prosthesis for one bone is not necessarily the optimal one for another bone. Finally, the fourth problem, of a similar kind, is related to the nature and complexity of the FEM model used in the optimization process, which also determines the outcome. Simplified, e.g. two- dimensional models have the obvious disadvantage of neglecting a number of structural aspects. 'Anatomic' FEM models, on the other hand, have the disadvantage of complexity, which makes them expensive and their results difficult to interpret and generalize (Huiskes and Chao, 1983).

In addition to these problems, it is obvious that design optimization can only be carried out relative to a specific design concept, which has to be determined first. With regard to the femoral hip component, for example, it must first be decided whether intramedullary cement or cementless fixation is used, whether or not a collar will be included, etc.

It is evident from these considerations, that design optimization is a rather narrow process, for which the conditions must be well defined. The present investigation was limited to the femoral hip stem, fixed with acrylic cement, without a proximal collar to rest on the calcar of the bone. The design objective was restricted to an optimization of the load-transfer process from stem to bone, minimizing the chances for cement/bone interface disruption (i) and acrylic cement failure (ii). To evaluate the effects of variable loading, it was recognized that the effect of the hipjoint force can be separated into bending, transverse and axial load-transfer, each of which was analysed separately in the simplified model. For reasons indicated earlier, pure bending was assumed in the twodimensional FEM model.

The one-dimensional FEM model applied particularly emphasizes the load-transfer mechanism and the stresses which are directly associated with that mechanism in a continuum model of similar geometrical simplicity (Huiskes, 1980). Such a model can be considered as a generalized conceptual representation of intramedullary fixation, whereby only the tendencies of the stress patterns, as they depend on structural properties, can be related to a real THA configuration (Huiskes, 1980). The two-dimensional FEM model with side-plate and nonuniform element thickness is more realistic, in the sense that its flexibility characteristics approximately reproduce a three-dimensional configuration which is symmetric relative to, and loaded in, the frontal plane; as, for example, the model used by Crowninshield et al. (1980). It must be noted that interface bonding was assumed in the models. In the case that interfaces are loose, or fibrous tissue interposition occurs between cement and bone, the load-transfer mechanism may change drastically (Weinans et al., 1988).

The shape optimization scheme used here is relatively simple, straightforward and applicable in principle for all FEM configurations, but requires much computer time. For the present problems, the least-pth search procedure was found to be the most efficient and effective, relative to other methods mentioned above. The effectiveness of this method is related to the particular goal of uniform stress distributions, where peak values are to be penalized. The efficiency is improved by gradually increasing the value of the 
exponent $p$. Still, the determination of the search direction takes multiple iterations to the extent that on the order of $100 \mathrm{FEM}$ calculations may be required for a complete optimization procedure. Sensitivity analysis methods such as suggested by Yang et al. (1984) could maybe serve to improve the efficiency of the optimization scheme (Yoon et al., in press)

The optimization analyses showed that the optimal stem shape for the objective criteria selected here is consistent in its general characteristics for different kinds of loading, structural properties and FEM models, featuring proximal and distal tapers, and a belly-shaped mid-region. In an extension of the present project it was found that alternative objective functions, minimizing interface shear and tensile stresses, yielded similar shapes (Huiskes and Boeklagen, 1988). Relative to 'traditional' stem shapes, and depending on the loads and properties, the optimal stem may produce cement and cement/interface stress reductions in the range of $30-70 \%$.

Although consistent in its general characteristics, the dimensional details of the optimal shape depend on the stem length, and on the elastic moduli of stem, bone and cement. The parametric analysis showed that to obtain maximal stress reductions, the stem should not be extremely long, nor extremely short, and preferably made out of a CoCr-alloy, rather than titanium.

A final word must be devoted to the actual realization of optimized stem shapes in practice. Evidently, the shape of a prosthesis will not be dictated by the outcome of an optimization process, because other considerations (e.g., surgical technique, possibilities for removal, manufacturing techniques) will have to play a role as well. For the shape obtained in this study, for instance, a special insertion technique would have to be used to ensure proper alignment of the prosthesis relative to the bone. In addition, patient bones are not identical in geometry and properties, hence, unless a customized approach was used, the actual prosthetic design would have to be some sort of compromise, representing an adequate solution for a class of bones. Evidently, the mechanically optimized shape can only serve as a guideline in a design process.

Acknowledgements - Sponsored in part by Orthopaedic Technology BV, The Netherlands, and by the M. E. Müller Foundation, Bern, Switzerland.

\section{REFERENCES}

Crowninshield, R. D., Brand, R. A., Johnston, R. C. and Milroy, J. C. (1980) An analysis of femoral component stem design in total hip arthroplasty. $J$. Bone $J t$ Surg. $62 \mathrm{~A}$ 68-78.

Crowninshield, R. D., Johnston, R. C. and Andrews, J. G. (1978) A biomechanical investigation of the human hip. J. Biomechanics 11, 75-86.

Daniels, R. W. (1978) An Introduction to Numerical Methods and Optimization Techniques. Elsevier North-Holland, New York.

Davy, D. T., Kotzar, G. M., Brown, R. H.; Heiple, K. G. Goldberg, V. M., Heiple, K. G., Jr, Berilla, J. and Burstein, A. H. (1988) Telemetric force measurements across the hip after total arthroplasty. $J$. Bone $J t$ Surg. 70A, 45-50.

Huiskes, R. (1980) Some fundamental aspects of human-joint replacement. Acta orthop. scand. Suppl. 185.

Huiskes, R. (1986) Biomechanics of bone-implant interactions. Frontiers in Biomechanics (Edited by SchmidtSchönbein, G. W., Woo, S. L-Y. and Zweifach, B. W.) pp. 245-262. Springer, New York.

Huiskes, R. (1988) Stress patterns, failure modes and bone remodelling. Non-cemented Total Hip Arthroplasty (Edited by Fitzgerald, R. H., Jr) pp. 283-302. Raven Press, New York.

Huiskes, R. and Chao, E. Y-S. (1983) A survey of finite element methods in orthopaedic biomechanics: the first decade. J. Biomechanics 16, 385-409.

Huiskes, R., Janssen, J. D. and Slooff, T. J. (1981) A detailed comparison of experimental and theoretical stressanalyses of a human femur. Mechanical Properties of Bone (Edited by Cowin, S. C.), AMD-Vol. 45, pp. 211-234. The American Society of Mechanical Engineers, New York.

Huiskes, R. and Schouten, R. Y. (1980) The effect of interface loosening on the stress distribution in intramedullary fixated artificial joints. 1980 Advances in Bioengineering (Edited by Mow, V. C.), pp. 213-217. The American Society of Mechanical Engineers, New York.

Huiskes, R., Weinans, H., Grootenboer, H. J., Dalstra, M. Fudala, B. and Slooff, T. J. (1987) Adaptive baneremodeling theory applied to prosthetic-design analysis. $J$. Biomechanics 20, 1135-1151.

Huiskes, R. and Boeklagen, R. (1988) The application of numerical shape optimization to artificial-joint design. Computational Methods in Bioengineering (Edited by Spilker, R. L. and Simon, B. R.) BED-Vol. 9, pp. 185-197. The Mechanical Society of Mechanical Engineers, New York.

Stauffer, R. N. (1982) Ten-year follow-up study of total hip replacement. J. Bone Jt Surg. 64A, 983-990.

Weinans, H., Huiskes, R. and Grootenboer, H. J. (1988) The mechanical effects of fibrous tissue interposition at the cement-bone interface in THA. Transactions 34th Annual Meeting Orthopaedic Research Society, p. 502.

Yang, R. J., Choi, K. K., Crowninshield, R. D. and Brand, R. A. (1984) Design sensitivity analysis, a new method for implant design and a comparison with parametric finite element analysis. J. Biomechanics 17, 849-854.

Yoon, Y. S., Jang, G. H. and Kim, Y. Y. (in press) Shape optimal design of the stem of a cemented hip prosthesis to minimize stress concentration in the cement layer. $J$. Biomechanics. 